“ (C) 2019 IEEE. Personal use of this material is permitted. Permission from IEEE must be obtained for all other uses, in any current or future media, including

reprinting/republishing this material for advertising or promotional purposes, creating new collective works, for resale or redistribution to servers or lists, or reuse of any copyrighted component of this work in other works." 


\title{
A Wideband Low-Profile Tightly Coupled Antenna Array with a Very High Figure of Merit
}

\author{
Alpha O. Bah, Student Member, IEEE, Pei-Yuan Qin, Member, IEEE, Richard W. Ziolkowski, Fellow, \\ IEEE, Y. Jay Guo, Fellow, IEEE, and Trevor S. Bird, Fellow, IEEE
}

\begin{abstract}
A wideband, low-profile, tightly coupled antenna array with a simple feed network is presented. The dipole and feed network in each unit cell are printed on both sides of a single RT/Duroid ${ }^{\mathrm{TM}} 6010$ substrate with a relative dielectric constant of 10.2. The feed network, composed of meandered impedance transformer and balun sections, is designed based on Klopfenstein tapered microstrip lines. The wide angle impedance matching is empowered by a novel wideband metasurface superstrate. For the optimum design, scanning to $70^{\circ}$ along the E-plane is obtained together with a very high array figure of merit, $P_{A}=2.84$. The H-plane scan extends to $55^{\circ}$. The broadside impedance bandwidth is 5.5:1 (0.80 - 4.38) GHz with an active VSWR value $\leq 2$. The overall height of the array above the ground plane is $0.088 \lambda_{\mathrm{L}}$, where $\lambda_{L}$ is the wavelength at the lowest frequency of operation. A prototype was fabricated and tested to confirm the design concepts.
\end{abstract}

Index Terms-Bandwidth, metasurface, phased arrays, tightly coupled arrays, wide angle impedance matching, ultrawideband arrays, figure of merit.

\section{INTRODUCTION}

$\mathrm{R}$ ECENT advances in radar, communications and sensing, combined with the shrinking size of antenna platforms, have highlighted the need for wideband multifunctional phased arrays [1, 2]. These wideband arrays can produce multiple beams, polarizations, and frequency bands. They can consolidate various systems onto a single wideband device ensuring substantial reduction in size, weight, cost and power consumption [3].

There have been numerous investigations into increasing antenna array bandwidth using a wide variety of techniques. One such technique uses the Vivaldi antenna to achieve over a 10:1 impedance bandwidth $[4,5]$. However, these antennas are usually heavy, are wide spread in the E-plane, have high cross-polarization levels in the intercardinal planes, and can be

Manuscript received September 4, 2018; accepted October 16, 2018. Date of publication January 11, 2019; date of current version March 9, 2019. This work is supported by the Australian Government Research Training Program Scholarship, the Cooperate Research Centre for Space Environment Management (SERC Limited), and the Australia Research Council Discovery Program under Grant DE170101203 and Grant DP160102219. The authors are with the Global Big Data Technologies Centre, University of Technology Sydney, New South Wales, 2007, Australia (e-mail: alpha.bah@student.uts.edu.au; peiyuan.qin@uts.edu.au; jay.guo@uts.edu.au; richard.ziolkowski@uts.edu.au)

Color versions of one or more of the figures in this communication are available online at http://ieeexplore.ieee.org.

Digital Object Identifier 10.1109/TAP.2015.????? several wavelengths thick [6]. The modified Vivaldi (i.e. balanced antipodal Vivaldi antenna, BAVA) [7] can achieve a 10:1 bandwidth with a $\lambda_{\mathrm{H}} / 2$ thick profile and has a better than $-17 \mathrm{~dB}$ cross-polarization level across all planes where $\lambda_{\mathrm{H}}$ is the wavelength at the highest frequency of operation. Nonetheless,

the BAVA shows high scanning voltage standing wave ratios (VSWR) across the H-plane.

Tightly coupled antenna arrays (TCAA) [8-11] are another class of periodically fed wideband arrays. Their elements can either be capacitively coupled $[8,9]$ or directly connected $[10$, 11] to each other. One major challenge in TCAAs is the design of an equally wideband feed network. The earlier prototypes [8] made use of bulky feed organizers and commercially available external $180^{\circ}$ hybrids for cable routing, common mode (CM) suppression, and balanced feeding. Subsequent TCAA designs have attempted to incorporate the feed network into the array design in order to remove the feed organiser. The tightly coupled dipole array with integrated balun feeds (TCDA-IB) $[9,12]$ helped overcome the size, weight, and bandwidth limitation of previous feeds. They facilitated bandwidths up to 7.35:1. Another type of wide band array is the planar ultra-wideband modular array (PUMA) $[13,14]$. The PUMA array uses shorting posts to mitigate $\mathrm{CM}$ resonances. Bandwidths up to $6: 1(7.5-45 \mathrm{GHz})$ have been obtained with low VSWR values while scanning to $45^{\circ}$. Perforations were made on the thick antenna substrate to reduce its effective dielectric constant and to help control the amount of surface waves.

Another major challenge in TCAA design is how to compensate for the increased scan loss due to the impedance mismatch at the aperture-air interface [15]. To address this problem, wide angle impedance matching (WAIM) superstrates have been employed. Dielectric WAIMs [16, 17] can operate over a wide bandwidth, although the scanning improvement achieved is not uniform across all planes. The use of thicker superstrates to achieve a better wideband match also increases the overall weight of the array and increases the chance of scan blindness. More recently, metasuraface (MS) $[18,19]$ and FSS [20] WAIMs have been used to alleviate the weight and volume problems of dielectric WAIMs and to improve the achievable scan angles. However, the achievable bandwidth for the MS WAIMS have been quite narrow and the vertically oriented FSS elements increase the antenna profile.

In this paper, we report a new wideband TCAA feed structure and a novel wideband MS-WAIM superstrate to 
address the aforementioned two challenges. The feed is a single layer, low-profile structure with combined impedance transformer and balun functionalities. It can provide more than 6:1 impedance bandwidth while maintaining a high level of current balance. The MS-WAIM, used to improve the scan performance over a wide bandwidth, is composed of tightly-coupled unequal arm Jerusalem cross (TC-UAJC) elements printed on its two sides. Interestingly, the MS-WAIM also improves the cross-polarization levels as the scan angle increases. It is based on a single sided (SS) MS-WAIM first introduced by the authors [21]. Compared to [21], substantial additional contributions are made in this paper. Firstly, the design in this work introduces a realistic wideband feed network which also helps to lower the array profile rather than the ideal lumped port feed used in [21]. Secondly, detailed array analysis and design guidelines are presented for two thicknesses of the MS-WAIM. Thirdly, a fully characterized 10 $\mathrm{x} 10$ array prototype is presented that validates the design concepts and simulation methodology employed in this work. Finally, wide scan angles of up to $70^{\circ}$ along the E-plane are demonstrated.

An array figure of merit $\left(\mathrm{P}_{\mathrm{A}}\right)$ [22] was calculated for the optimum TCAA design, to make comparisons with similar designs in the literature. The figure of merit value obtained, $\mathrm{P}_{\mathrm{A}}$ $=2.84$, which is higher than most of the designs reported to date. The array profile is only $0.088 \lambda_{\mathrm{L}}$, where $\lambda_{\mathrm{L}}$ is the wavelength at the lowest frequency of operation. It can scan to $70^{\circ}$ along the E-plane and $55^{\circ}$ along the $\mathrm{H}$-plane, with an active VSWR $\leq 3.2$. The broadside impedance bandwidth is $5.5: 1$ $(0.80-4.38) \mathrm{GHz}$. The improved scan performance was obtained by optimizing the array dimensions for both broadside and at the widest scan angles.

The paper is organized as follows. Section II is devoted to the tightly coupled array unit cell design that incorporates the feed network and metasurface. The radiation performance of the computed finite array and manufactured prototype are presented in Section III. Sections IV and V present the discussion and implications of our results. Finally, in Section VI, our conclusions are presented.

\section{Tightly COUPLED ANTENNA ARRAY DESIGN}

In this section, the design for the constituent parts of the TCAA shown in Fig. 1 will be discussed. A unit cell design of an overlapped dipole antenna in an infinite array setting with a $170 \Omega$ lumped port feed is first presented. This is followed by the design of a double sided (DS) MS-WAIM and the extraction of its constituent parameters. Next, the design of a realistic wideband balun to feed the array is presented. Finally, following the introduction of a balun in the array, methods are devised to suppress the CM currents that arise, to improve the range of scanning angles in the elevation plane, and to reduce the levels of cross-polarization for the designs presented.

\section{A. Lumped Port Fed Unit Cell Design}

An expanded view of the lumped port fed dipole (rotated to fit) is shown in Fig. 1 (b). In the preliminary simulations, the TCAA unit cell contained an overlapped dipole antenna fed with a $170 \Omega$ lumped port impedance placed at a distance of $h_{\text {gnd }}$ $=28.2473 \mathrm{~mm}$ above a PEC ground plane. The dipole is

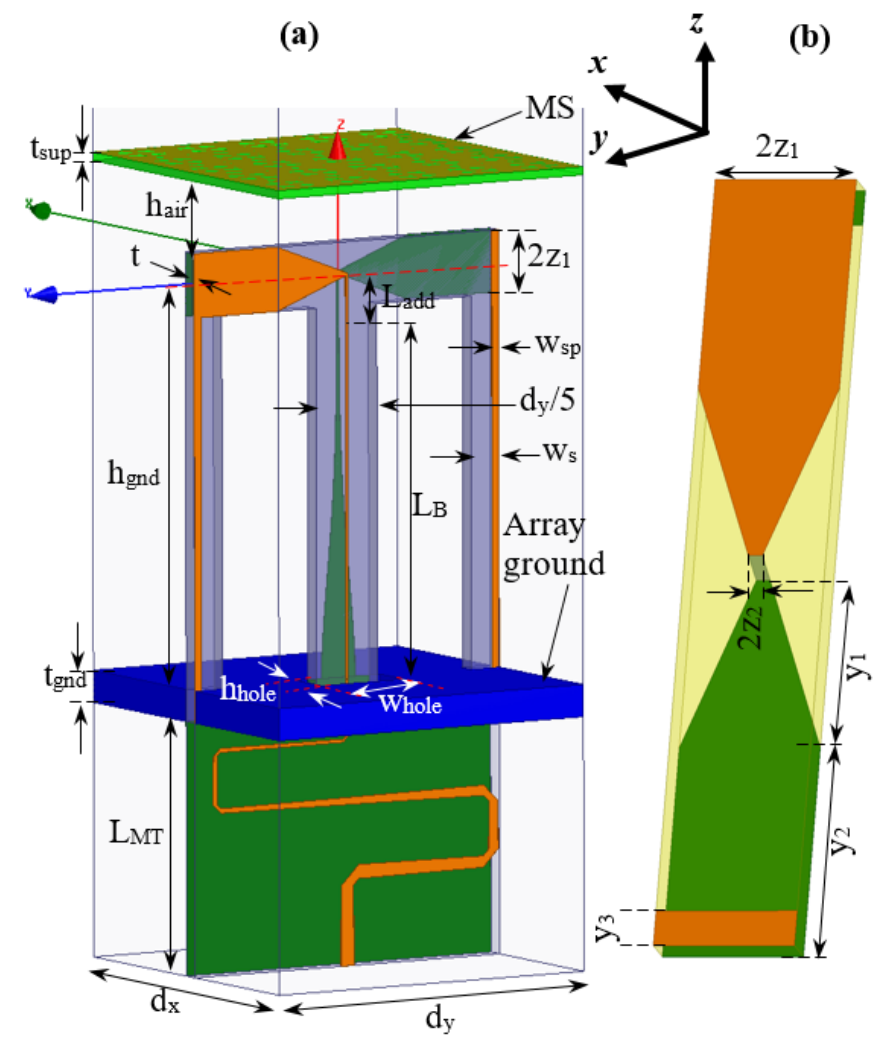

(c)

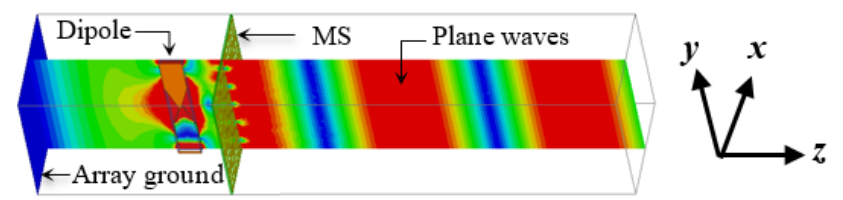

Fig. 1. Infinite TCAA. (a) Perspective view of the balun fed TCAA unit cell with a MS-WAIM superstrate, shorting pins and a perforated feed substrate. The feed and dipole were designed on a Rogers RT/Duroid ${ }^{\mathrm{TM}} 6010$ substrate with a thickness, $\mathrm{t}=1.016 \mathrm{~mm}$ and relative dielectric constant, $\varepsilon_{\mathrm{r}}=10.2$. (b) Expanded view of the lumped port fed dipole. (c) Electric fields along the $y-z$ plane for the lumped port fed unit cell.

oriented vertically along the $y-z$ plane with its arms printed on opposite sides of a RT/Duroid ${ }^{\mathrm{TM}} 6010$ substrate with a thickness, $\mathrm{t}=1.016 \mathrm{~mm}$ and relative dielectric constant, $\varepsilon_{\mathrm{r}}=$ 10.2. The period of the unit cell is $d_{x}=d_{y}=24 \mathrm{~mm}$. The infinite array is created using Floquet mode analysis plus master-slave periodic boundary conditions along the $x$ and $y$ directions. To achieve a wideband impedance match for the dipole array, the feed flared section, $\mathrm{y}_{1}(4 \mathrm{~mm})$, the length of the dipole arms, $\mathrm{y}_{2}$ $(8 \mathrm{~mm})$, the capacitive coupling between adjacent elements, $\mathrm{y}_{3}$ $(1 \mathrm{~mm})$, the arm widths, $2 \mathrm{z}_{1}(4 \mathrm{~mm})$, and the width of the lumped port, $2 \mathrm{z}_{2}(0.12 \mathrm{~mm})$ were all tuned. The dipole antenna is covered with a 5 x 5 array of the MS-WAIM at a height of $h_{\text {air }}$ $=6.761 \mathrm{~mm}$. The overall height of the array from the ground plane to the top of the MS is $37.2623 \mathrm{~mm}$.

The impedance bandwidth of the lumped port fed infinite dipole array at broadside for VSWR $\leq 2$ is $6.23: 1(0.79 \mathrm{GHz}-$ $4.92 \mathrm{GHz}$ ), which will be discussed in the next sub-section. 


\section{B. Double Sided MS-WAIM Design}

The proposed double sided metasurface (DS-MS) unit cell is depicted in Fig. 2. It is composed of tightly-coupled unequal arm Jerusalem cross (TC-UAJC) elements printed on the two sides of a Rogers 5880 substrate. The bottom element is rotated $90^{\circ}$ relative to the element at the top to ensure uniform performance when excited along the $\pm \mathrm{z}$ directions. The effective medium parameters of the MS-WAIM were extracted from its reflected and transmitted signals using the method described in [23]. These extracted parameters are shown in Fig. 3 . It is seen that $\mu_{\text {eff }}$ is zero and $\varepsilon_{\text {eff }}$ has a constant value of 8.6 within the frequency range of interest (0 to $5.0 \mathrm{GHz}$ ). A mu zero material has a zero index of refraction. According to Snell's law, the angle of refraction from this type of material is zero degrees irrespective of the angle of incidence within the material. As a result, the DS-MS is able to transform the cylindrical waves from the array into plane waves with minimal phase variation to enable wide angle scanning. This waveform transformation can also help to lower the array profile since all the energy is concentrated in the forward direction. This phenomenon is demonstrated in Fig. 1 (c).

The MS-WAIM also introduce a capacitive reactance to counteract the effects of the array's inductively reactive ground plane thereby minimizing the variation of the array's active reflection coefficients at wider scan angles. The solid red curve in Fig. 4. Shows the effectiveness of the MS-WAIM as a wideband (6.23:1) impedance matching layer. Without the MS-WAIM, the array is highly unmatched across most of the band.

When the MS structure is integrated with the TCAA, its dimensions were adjusted to compensate for the near field interactions. The final optimized MS dimensions were: $r_{1}=$ $1.59 \mathrm{~mm}, \mathrm{~g}_{1}=0.9774 \mathrm{~mm}, \mathrm{~g}_{2}=0.2384 \mathrm{~mm}$, and $\mathrm{L}_{2}=4.4568$ $\mathrm{mm}$ with the other dimensions as shown under Fig. 2.

\section{Balun Design}

To cover the inherent wide bandwidth of the TCAA, an equally wideband feed network is required. The proposed feed network consists of an impedance transformer and a balun (B), both of which were constructed from Klopfenstein [24] tapered microstrip lines. The feed network was designed on the same substrate type as the TCAA and for a lower cut off frequency of $0.5 \mathrm{GHz}$. The Klopfenstein equations given in [24] were evaluated using MATLAB [25] to obtain the impedance and reflection coefficient variation with distance along the taper. The closed form synthesis equations given in [26] were then used to calculate the physical dimensions of the taper width for all the impedance values. The resulting dimensions were then imported into the commercially available software, ANSYS High Frequency Structure Simulator, ANSYS-HFSS [27], to model and optimize the design.

The straight impedance transformer (ST) was designed for an input impedance of $50 \Omega$ on one end and an impedance of $92.356 \Omega$ on the output. The latter corresponds to a line width of $0.1551 \mathrm{~mm}$ on the microstrip lines, which in turn gives an impedance of $150 \Omega$ on the parallel strip lines.

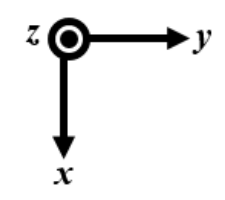

(a)

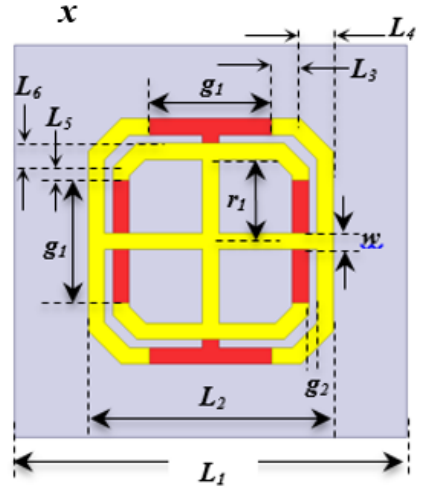

(b)

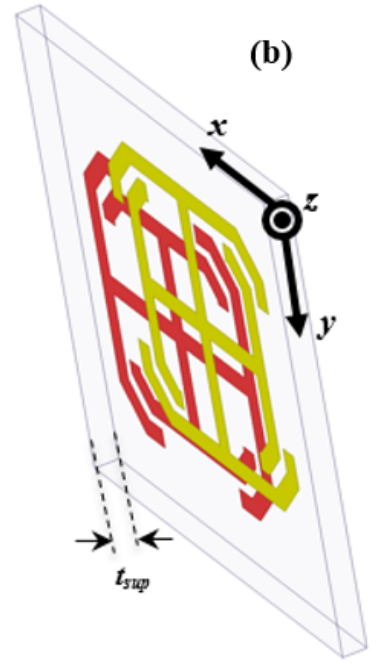

Fig. 2. DS-MS unit cell geometry. (a) Top view. (b) Perspective view. The DS-MS unit cell dimensions in millimeters are: $\mathrm{w}=0.2, \mathrm{t}_{\text {sup }}=0.254, \mathrm{~g}_{1}=1.5, \mathrm{~g}_{2}$ $=0.1, \mathrm{~L}_{1}=4.8, \mathrm{~L}_{2}=3.0, \mathrm{~L}_{3}=0.35, \mathrm{~L}_{4}=0.4, \mathrm{~L}_{5}=0.15, \mathrm{~L}_{6}=0.3$, and $\mathrm{r}_{1}=1.0$. The substrate is Rogers RT/Duroid ${ }^{\mathrm{TM}} 5880$ with $\varepsilon_{\mathrm{r}}=2.2$ and $\tan \delta=0.0009$. The direction of propagation of the exciting wave is along the $\mathrm{z}$-axis.

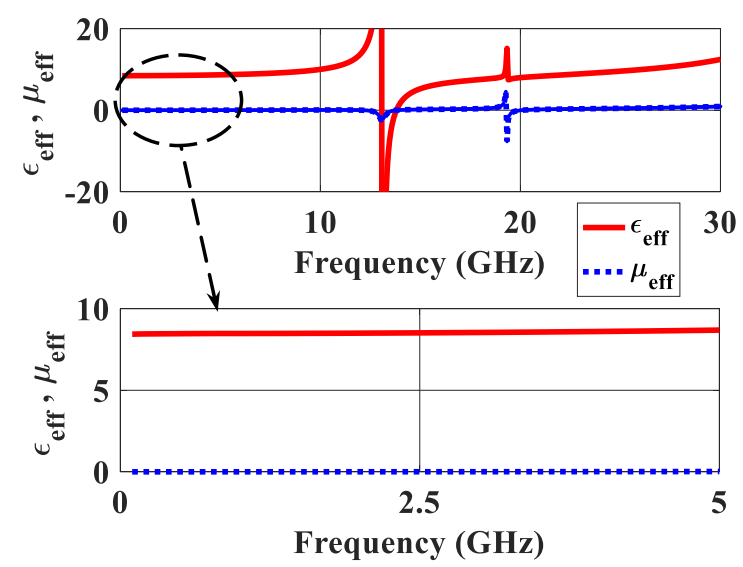

Fig. 3. The extracted $\varepsilon_{\text {eff }}$ and $\mu_{\text {eff }}$ for the DS-MS.

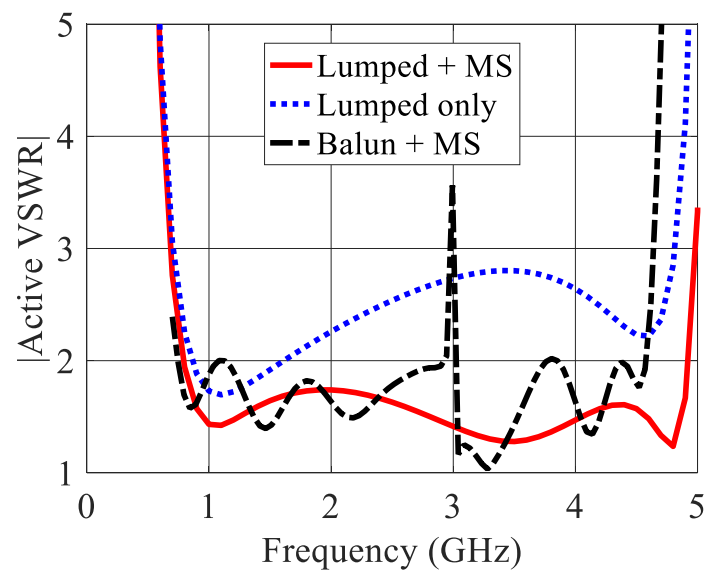

Fig. 4. Active VSWR of the infinite dipole array at broadside. The solid-red curve represents the lumped port fed array with the MS-WAIM superstrate. A 6.23:1 impedance bandwidth $(0.79 \mathrm{GHz}-4.92 \mathrm{GHz})$ is obtained (solid-red curve). The dotted-blue curve represents the lumped port fed array with no superstrate. The dashed-black curve represents the balun fed array with the MS-WAIM superstrate but without any shorting pins. 
The lower $150 \Omega$ impedance was chosen instead of an initial lumped port impedance of $170 \Omega$ since the corresponding line width would be too narrow to be manufactured accurately. The length of this transformer, $\mathrm{L}_{\mathrm{ST}}$, was found to be $50.5368 \mathrm{~mm}$. To reduce the profile of the array, the transformer was meandered using the optimum miter formulation [28]. During this design, it was discovered that using the optimum mitre bends, resulted in extremely thin bends. To overcome this problem, both the inner and outer corners of the bends were mitered by equal amounts which gave rise to bends with more surface area. These mitered bends are shown as solid black in Fig. 5. Also shown in Fig. 5 is an additional solid black rectangular pad of length $\mathrm{W}_{\text {add }}=2 \mathrm{~mm}$ at the commencement of the trace. This pad was added to act as a connection point of the SMA connector, which was included in the simulation to produce realistic results. The meandered version of the ST is indicated by the $\mathrm{L}_{\mathrm{MT}}$ section in Fig. 5. To produce a balanced feed for the TCAA, the ground plane at the $92.356 \Omega$ port of the unbalanced transformer was gradually tapered to a balanced parallel strip of equal width with the top trace. The width of the top trace of the balun, $\mathrm{W}_{\text {out }}$, remained uniform along its length. The balun section of the feed structure is indicated by $L_{B}$ in Fig. 5 . The full and tapered sections of the ground plane are shown in green in Fig. 5.

The simulated magnitude of the reflection coefficient of the meandered transformer and balun (MT and B), balun (B), straight transformer and balun (ST and B), and straight transformer (ST) are shown in Fig. 6. The reflection coefficient for all four cases are $<-13 \mathrm{~dB}$ from $0.5 \mathrm{GHz}$ to $4.5 \mathrm{GHz}$. We can see that in the "MT and B" cases, the high frequency performance deteriorates compared to the "ST and B" cases. This is due to increased reflections at the bends. Nevertheless, the "MT and B" performance is $<-13 \mathrm{~dB}$ within the frequency range of interest.

To find out the current balance on the feed structure, the ratio of the surface currents along a line for the top and bottom conductors was computed using HFSS. It can be seen from Fig. 7 that the currents on the last $(5-10) \mathrm{mm}$ sections of the B, MT and B, ST and B are practically identical indicating a high level of current balance within those sections. These portions of the traces are enclosed within the solid-blue, dotted-red, and dashed-black (Comment: change to account for B\&W only) ovals respectively. (Comment select hatchings in black \& white).

\section{Balun Fed Unit Cell Design}

A detailed view within the TCAA unit cell incorporating a meandered wideband balun is shown in Fig. 1 (a). The E-plane is along the $y-z$ direction and the H-plane is along the $x-z$ direction.

Following the successful design of the MS, the lumped port fed antenna array, and the wideband balun, the next step was to integrate all the parts and perform an overall optimization. Several adjustments were made to bring the whole antenna inside the unit cell into balance. A variable length of transmission line, $\mathrm{L}_{\text {add }}$, of equal width was added to the tips of the top and bottom conductors of the balun.

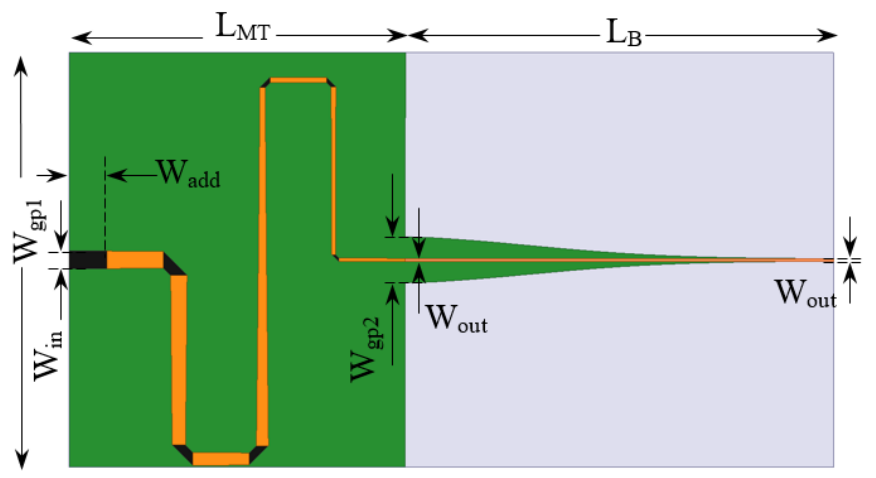

Fig. 5. Top view of the meandered impedance transformer (MT) and balun (B). The optimized balun dimensions are: $\mathrm{W}_{\mathrm{in}}=0.9279 \mathrm{~mm}, \mathrm{~W}_{\text {out }}=0.1551 \mathrm{~mm} \mathrm{~W} \mathrm{gp}_{1}$ $=\mathrm{d}_{\mathrm{y}}=22.0 \mathrm{~mm}, \mathrm{~W}_{\mathrm{gp} 2}=2.1048 \mathrm{~mm}, \mathrm{~W}_{\text {add }}=2.0 \mathrm{~mm}, \mathrm{~L}_{\mathrm{MT}}=15.8417 \mathrm{~mm}, \mathrm{~L}_{\mathrm{B}}=$ $25.2413 \mathrm{~mm}$.

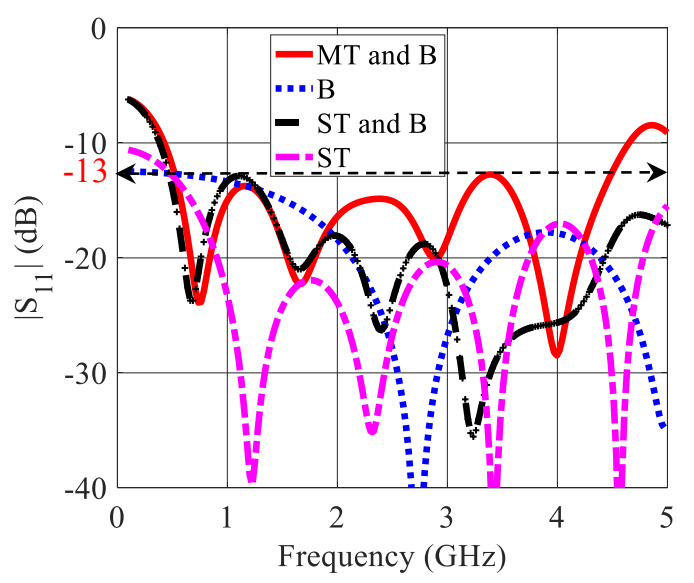

Fig. 6. Magnitude of the reflection coefficient of the meandered transformer and balun (MT and B), balun (B), straight transformer and balun (ST and B), and straight transformer (ST).

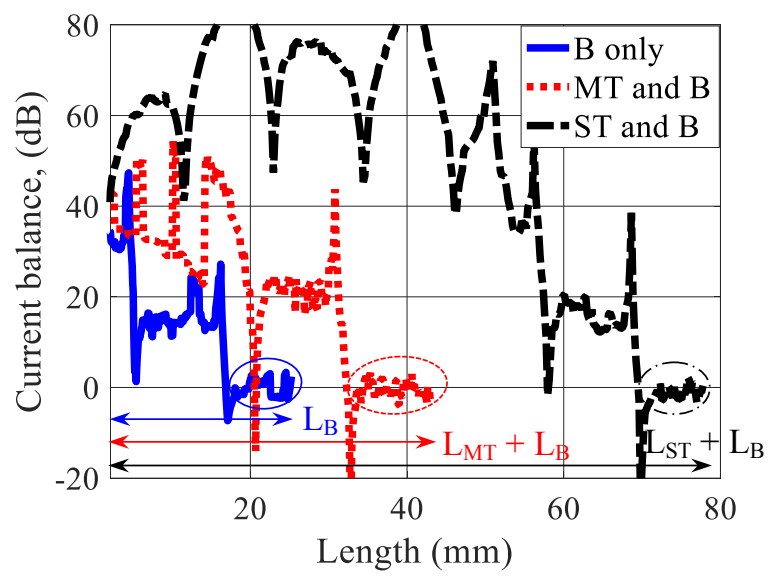

Fig. 7. Magnitude of the line current balance along the axis of the balun (B), meandered transformer and balun (MT and B), and straight transformer and balun (ST and B). The portions of the traces within the ovals is where the line currents on the top and bottom conductors are practically identical.

This additional length of line was used to equalize the antenna input impedance to the characteristic impedance of the balun. 


\section{1) Common Mode Suppression}

Although the stand-alone balun was well matched, when placed in the array setting, the strong mutual coupling between adjacent units causes the feed to be unbalanced. These unbalanced currents led to an induced common-mode resonance around mid-band (see dashed curve in Fig. 4) as was reported in [13] and a reduction in the achievable bandwidth. To eliminate these CM currents, the first step was to reduce the array unit cell size by reducing $\mathrm{y}_{1}$ from $6 \mathrm{~mm}$ to $5 \mathrm{~mm}$. The inner radius of the MS unit cell, $\mathrm{r}_{1}$, was also reduced from 1.59 $\mathrm{mm}$ to $1.4 \mathrm{~mm}$ to fit into the new array unit cell. In the consequent step, a shorting pin to ground was also introduced on the excited arm of the dipole. Finally, cuts were made on the feed substrate on either side of the balun to reduce the amount of coupling.

With the addition of the shorting pins, the $\mathrm{CM}$ resonance moved higher up in frequency but not entirely out of band. A parametric study was carried out on $\mathrm{L}_{\mathrm{add}}$, and the dipole overlap, $\mathrm{y}_{3}$. The result of this study is displayed in Fig 8. In Fig. 8 (a), when $\mathrm{L}_{\text {add }}$ is reduced from $4.0 \mathrm{~mm}$ to zero $\left(\mathrm{y}_{3}=1.0 \mathrm{~mm}\right)$, the $\mathrm{CM}$ resonance is moved almost entirely out of band, but the low frequency behavior of the array deteriorates. The shorting pin creates a resonance loop with the driven arm of the dipole and the array ground plane. By reducing the value of $\mathrm{L}_{\text {add }}$, the CM resonance is shifted to higher values and eventually out of band. This method has the added benefit of reducing the array height by $4 \mathrm{~mm}$ whilst ensuring a CM-resonance free array. To improve the low frequency behavior of the array, the optimum value of $L_{\text {add }}=0 \mathrm{~mm}$ found from the previous step was utilized. The value of $y_{3}$ was then varied from $0.5 \mathrm{~mm}$ to $2.0 \mathrm{~mm}$ and the result is displayed in Fig. 8 (b). For $\mathrm{y}_{3}=0.5 \mathrm{~mm}$, the low frequency behavior was restored, and the infinite array was matched over a slightly reduced impedance bandwidth of 5.58:1 $(0.77 \mathrm{GHz}-4.3 \mathrm{GHz})$ compared to the lumped port fed design.

\section{2) Common Mode Resonance Free Design}

In the previous case, $\mathrm{L}_{\text {add }}$ was decreased to zero to match the array and reduce the array profile in the process. To further reduce the array profile and to provide more flexibility in matching the array over wider scan angles, the balun was redesigned as was described earlier but with a reduced height of $22.7145 \mathrm{~mm}$. The impedance transformer section remained unaltered. This balun is henceforth referred to as the shorter balun (SB) throughout this paper. The rest of the antenna dimensions are given in Table I. With $\mathrm{L}_{\text {add }}=2 \mathrm{~mm}$ and $h_{\text {air }}=$ $5 \mathrm{~mm}$, scans of up to $70^{\circ}$ along the E-plane and $55^{\circ}$ along the H-plane were obtained over a maximum VSWR value of 3.15 as shown in Fig. 9 (a). The broadside impedance bandwidth is 5.5:1. The co- and cross-polarization discrimination is $>20 \mathrm{~dB}$ across the whole band as can be seen in Fig. 9 (b). In this work, Ludwig's third definition of cross-polarization [29] is utilized.

When scanning to wider angles, we need to find the effect on the cross-polarization levels. In Fig. 10 (a), we see that the Eplane cross-polarization levels around $\mathrm{f}_{\text {mid }}$ and $\mathrm{f}_{\mathrm{H}}$ improves as the scan angle increases. Around $\mathrm{f}_{\mathrm{L}}$, the increase in the cross-polarization levels while scanning from broadside to $70^{\circ}$ is only $3 \mathrm{~dB}$. The parameters $\mathrm{f}_{\mathrm{L}}, \mathrm{f}_{\text {mid }}$, and $\mathrm{f}_{\mathrm{H}}$ refers to the low frequency, mid frequency, and high frequency regions of the band

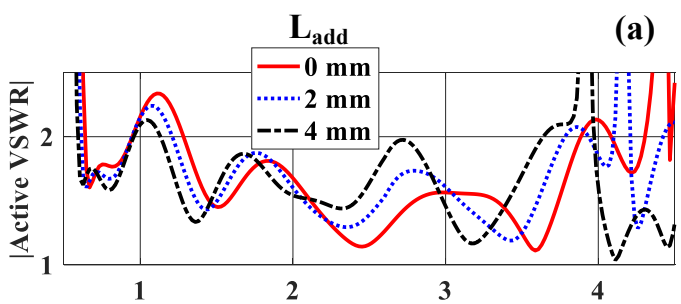

(b)

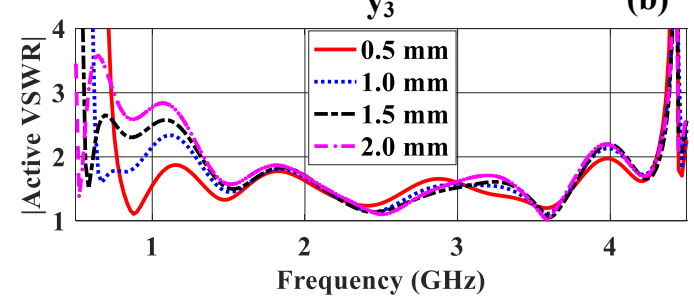

Fig. 8. Parametric study on the CM resolution process for the infinite array. (a) The additional length of transmission line, $\mathrm{L}_{\text {add }}$. (b) The dipole overlap, $\mathrm{y}_{3}$. A 5.58:1 broadside impedance bandwidth $(0.77 \mathrm{GHz}-4.3 \mathrm{GHz})$ was obtained.

(a)
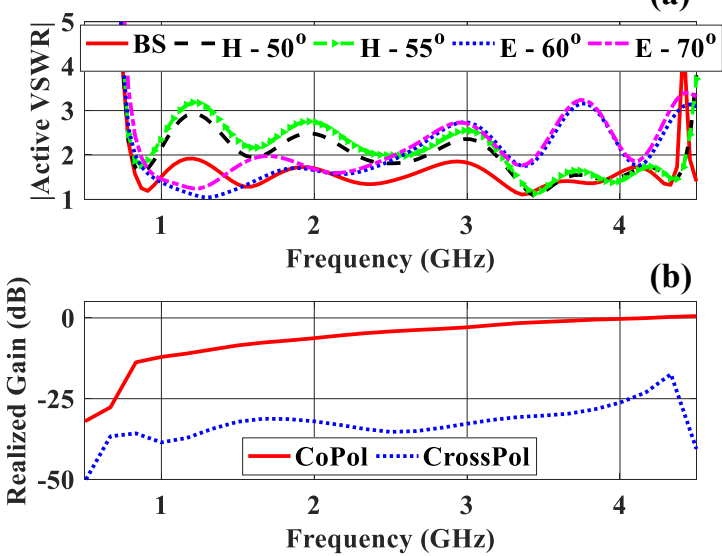

Fig. 9. The infinite array performance using the DS-MS $(0.254 \mathrm{~mm}$ thick) superstrate and the shorter balun feed. (a) Scanning ability. (b) Co- and cross-polarized realized gains.
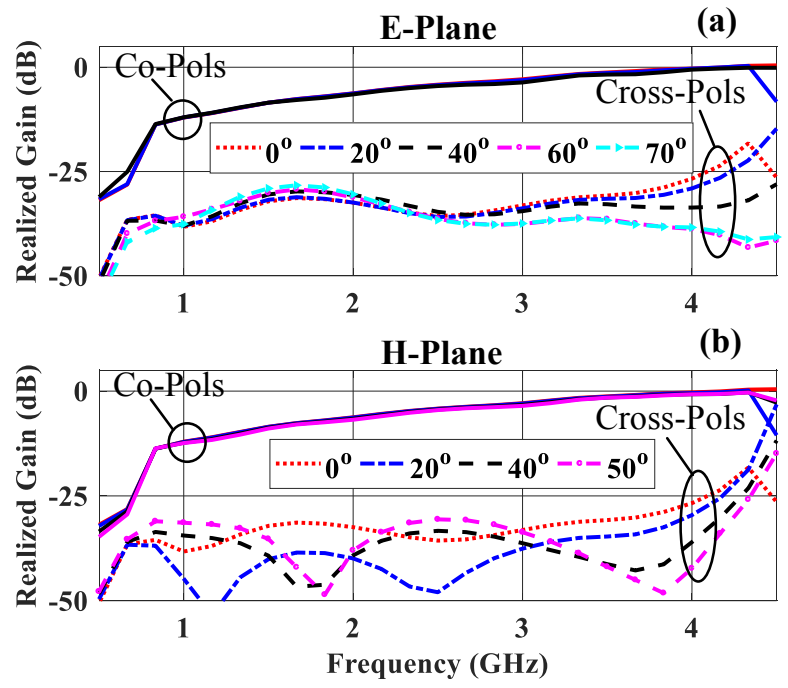
Fig. 10. The Co- and cross-polarization variation with frequency for various scan angles of the infinite array using the DS-MS (0.254 mm thick) superstrate and the shorter balun feed. (a) E-plane scan. (b) H-plane scan.

TABLE I

UNIT CELL DIMENSIONS (IN MM)

\begin{tabular}{ccccccccc}
\hline $\boldsymbol{t}_{\text {sup }}$ & $\boldsymbol{L}_{\text {add }}$ & $\boldsymbol{h}_{\text {air }}$ & $\boldsymbol{L}_{\boldsymbol{B}}$ & $\boldsymbol{w}_{\text {out }}$ & $\boldsymbol{y}_{\boldsymbol{1}}$ & $\boldsymbol{y}_{2}$ & $\boldsymbol{y}_{3}$ & $\boldsymbol{r}_{\boldsymbol{1}}$ \\
$0.254 \rightarrow$ & 2.0 & 5.0 & 22.71 & 0.1551 & 5.0 & 6.0 & 0.5 & 1.4 \\
$0.508 \rightarrow$ & 2.5 & 4.7 & 22.71 & 0.1551 & 5.0 & 6.0 & 0.5 & 1.4 \\
\hline \hline $\boldsymbol{z}_{1}$ & $\boldsymbol{w}_{\boldsymbol{s p}}$ & $\boldsymbol{w}_{\boldsymbol{s}}$ & $\boldsymbol{w}_{\text {hole }}$ & $\boldsymbol{h}_{\text {hole }}$ & $\boldsymbol{t}_{\text {gnd }}$ & $\boldsymbol{d}_{\boldsymbol{x}}$ & $\boldsymbol{d}_{\boldsymbol{v}}$ \\
$0.254 / 0.508 \rightarrow 2.0$ & 0.5 & 2.0 & 6.0 & 3.0 & 2.0 & 22.0 & 22.0 \\
\hline
\end{tabular}

TABLE II

ARRAY PERFORMANCE

\begin{tabular}{|c|c|c|c|c|c|}
\hline$t_{\text {sup }}(\mathrm{mm})$ & $\begin{array}{c}\text { Array height } \\
\text { (mm) }\end{array}$ & $\begin{array}{c}E-s c a \\
n\end{array}$ & $\begin{array}{c}H \text {-sc } \\
\text { an }\end{array}$ & $\begin{array}{l}\text { Range } \\
\text { (GHz) }\end{array}$ & Bandwidth \\
\hline $0.254 \rightarrow$ & -31.97 & $70^{\circ}$ & $55^{\circ}$ & $0.80-4.38$ & $5.48: 1$ \\
\hline $0.508 \rightarrow$ & -32.42 & $50^{\circ}$ & $55^{\circ}$ & $0.79-4.32$ & $5.47: 1$ \\
\hline
\end{tabular}

*Array height $=\mathbf{L}_{B}+\mathbf{L}_{\text {add }}+h_{\text {air }}+t_{\text {sup }}+\mathbf{z}_{1}$

respectively. The H-plane cross-polarization levels shown in Fig. 10 (b), improves across the whole band for the lower scan angles and across the $f_{H}$ region for all other angles. Around $f_{L}$ and $f_{\text {mid }}$, the cross-polarization levels are either comparable to or better than those at broadside. These are rather significant results as they indicate that this array can scan to wider angles without worrying about increasing cross-polarization levels. This phenomenon can be attributed to the MS-WAIM whose transmission phase variation with frequency for the TM polarized incident fields improves with increasing incidence angles [21].

The MS-WAIM discussed earlier is only $0.254 \mathrm{~mm}$ thick. This thickness was found to be too thin to hold its shape firmly above the array when constructed. A second array with a MS-WAIM thickness of $0.508 \mathrm{~mm}$ was designed with $\mathrm{L}_{\mathrm{add}}=$ $2.5 \mathrm{~mm}, \mathrm{~h}_{\text {air }}=4.7 \mathrm{~mm}$, and an overall array height of 32.4225 $\mathrm{mm}$ as shown in table I. The performance comparison of the two arrays is given in table II. The two arrays were found to have the same broadside impedance bandwidth but the second array was about $0.45 \mathrm{~mm}$ taller. It was discovered later that this small increase in height led to a reduction in the E-plane scan. As a proof of concept, the $0.508 \mathrm{~mm}$ MS was used during fabrication instead of the $0.254 \mathrm{~mm}$ one to minimise difficulties during assembly and measurement.

\section{RESULTS OF FABRICATED ARRAY}

A $10 \times 10$ prototype of the fabricated array utilizing the 0.508 mm MS-WAIM is depicted in Fig. 11. The elements along the first, second, ninth, and tenth columns were terminated by $50 \Omega$ loads to reduce the array edge effects. Only the edge elements along the E-plane were terminated given that the amount of mutual coupling is strongest along this plane and thus have a bigger impact on the edge effects. The active area of the array is the inner $6 \times 10$ elements. The electrical performance of this array was evaluated in terms of its matching and radiation characteristics.

\section{A. Antenna Construction}

The built array prototype and its constituent parts are shown in Fig. 11. The top and bottom of the fully assembled array is shown in Fig. 11 (a) and 11 (b) respectively. A block of expanded polystyrene foam $\left(\varepsilon_{\mathrm{r}}=1.03\right)$ was inserted into the array to help hold the antenna cards upright and to provide a

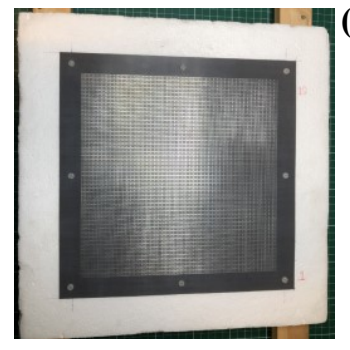

(a)

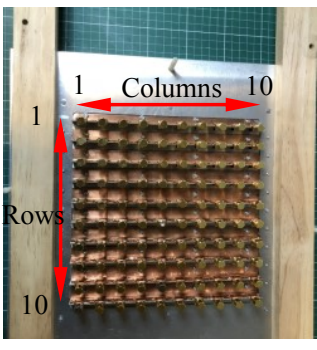

(b)

(c)

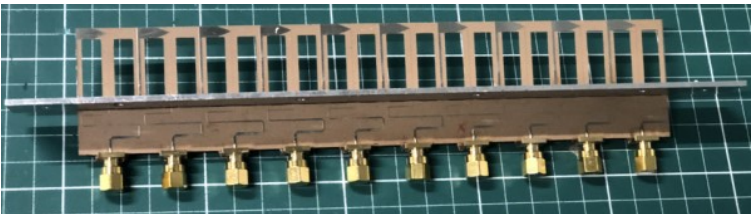

Fig. 11. Constituent parts of antenna prototype. (a) Top view of fully assembled array showing MS-WAIM and polystyrene foam. (b) Bottom view of fully assembled array with $50 \Omega$ terminations and the wooden planks used for mounting. (c) A sample antenna card containing 10 tightly coupled antennas attached to a strip of the inner portion of the aluminum ground plane.

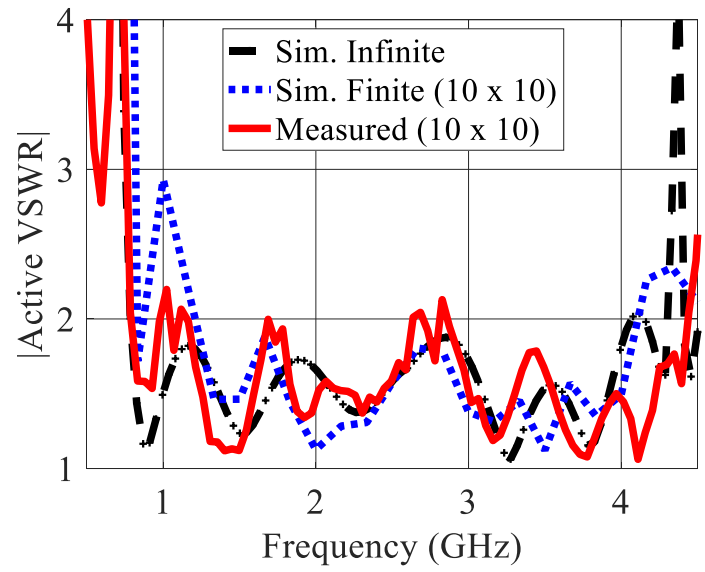

Fig. 12. The broadside active VSWR of the simulated infinite array, the simulated $10 \times 10$ finite array with 60 active elements, and the measured results obtained from the measured coupling coefficients.

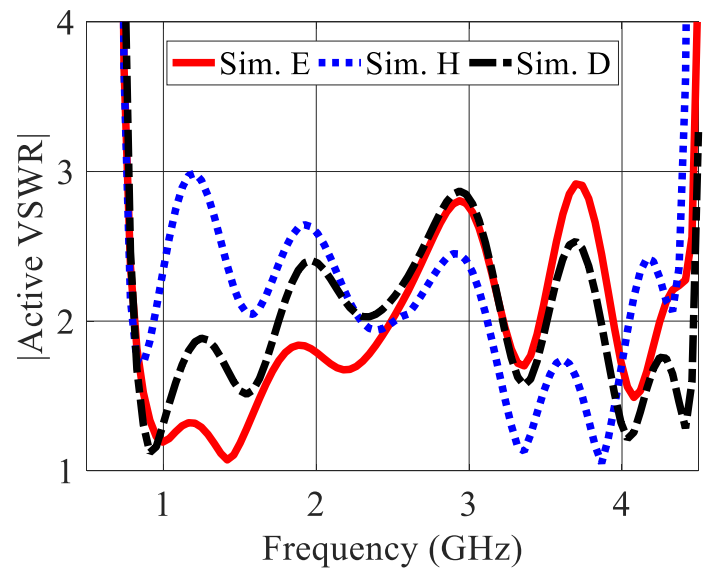


Fig. 13. The scanning active VSWR of the simulated infinite array across all three planes. Scan results shown are for $50^{\circ}$ along the E-plane, $55^{\circ}$ along the $\mathrm{H}$-plane and $65^{\circ}$ along the D-plane.

platform upon which the MS can rest. The MS was held in place by the foam platform and nine precisely cut plastic screws. Strips of copper tape was also used at the top of the array ground plane to enable soldering of the shorting pins from the dipole to the aluminium ground plane.

Below the array ground plane, ninety-degree copper brackets were manufactured and attached to the underside of the array to keep the antenna cards upright. One leg of the bracket was attached to the bottom surface of the array ground plane with metal screws while the other leg is attached to the ground plane portion of the antenna cards with copper tape and solder. Two long wooden planks, $40 \mathrm{~mm}$ wide, were attached to the bottom side of the array ground plane to act as the mounting points during testing.

The array aluminium ground plane is made up of an inner portion that is $2 \mathrm{~mm}$ thick and an outer cage that is $4 \mathrm{~mm}$ thick. The inner ground plane dimension is $264 \mathrm{~mm} \times 264 \mathrm{~mm}$. It was cut into strips to allow for the insertion of the antenna cards. Each of the ten antenna cards contains ten tightly coupled antenna elements attached to a strip of the inner ground plane as shown in Fig. 11 (c). A $2 \mathrm{~mm}$ deep by $22 \mathrm{~mm}$ wide portion was machined off on the inside perimeter of the outer ground plane to allow the inner ground plane to sit flush with the outer ground plane. The outer ground plane dimension is $352 \mathrm{~mm} \mathrm{x}$ $352 \mathrm{~mm}$.

\section{B. Active Matching Characteristics}

The active reflection coefficient of the built array was calculated from the measured coupling coefficients between the element in row 5 and column 5 (element 5,5 ) and every other element in the array. Measurements were carried out using a Keysight Technologies N5225A PNA. During each measurement period, the 98 non-active elements were terminated with $50 \Omega$ loads. The active reflection coefficient for element $i$, scanning in the $\left(\theta_{o}, \varphi_{o}\right)$ direction can be estimated using [30]:

$$
\begin{aligned}
& \Gamma_{a}\left(\theta_{o}, \varphi_{o}\right) \\
& =\sum_{n=1}^{N} S_{n i}\left|a_{n}\right| e^{-j k_{o}\left(x d_{x} \sin \theta_{o} \cos \varphi_{o}+y d_{y} \sin \theta_{o} \sin \varphi_{o}\right)}
\end{aligned}
$$

Where $S_{n i}$ is the measured complex coupling coefficients between element $i$ and the other elements of the array; $\left|a_{n}\right|$ is the magnitude of the excitation of the radiating element; $\mathrm{N}$ is the total number of elements; $k_{o}$ is the free space wave number; $x, y$ are the element numbers and $d_{x}, d_{y}$ are the element spacings along the $\boldsymbol{x}$ and $\boldsymbol{y}$ directions respectively.

The active VSWR was deduced from the active reflection coefficient for broadside and for scanning in the E, H, and D (diagonal) planes. The broadside active VSWR of the simulated infinite array, the simulated $10 \times 10$ finite array with 60 active elements, and the measured results obtained from the measured coupling coefficients are displayed in Fig. 12. There is a high degree of correlation between the three results across the band from $0.80 \mathrm{GHz}-4.38 \mathrm{GHz}$. The resonance around $4.4 \mathrm{GHz}$ in the infinite array is less pronounced in the finite array and measured results. There are some ripples on the measured results due to diffraction along the array edges and the finite

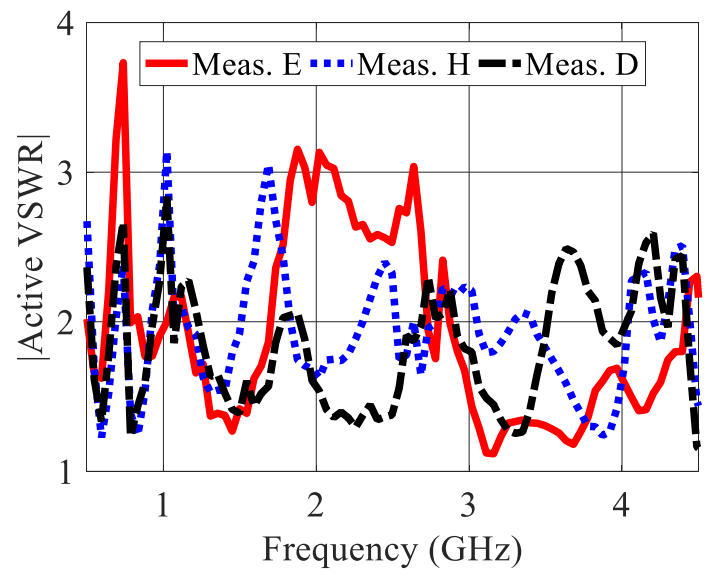

Fig. 14. The scanning active VSWR obtained from the measured coupling coefficients across all three planes. Scan results shown are for $50^{\circ}$ along the E-plane, $55^{\circ}$ along the H-plane and $65^{\circ}$ along the D-plane.

ground plane. The low frequency behavior of the simulated and measured finite arrays show reduced matching.due to the small number of active elements. Better matching can be obtained by using a larger sized array but with significantly higher computational over heads.

The simulated and measured scanning ability of the array is shown in Figs. 13 and 14 respectively. The plots for the measured results were obtained by using equation (1) above. The measured and simulated results agree well with each other for scan angles of up to $50^{\circ}$ along the E-plane, $55^{\circ}$ along the $\mathrm{H}$-plane and $65^{\circ}$ along the D-plane all under a VSWR value of 3.2. The array edge effects discussed earlier is manifest more pronouncedly along the E-plane during scan as an increase in the VSWR around mid-band.

\section{Radiation Characteristics}

The radiation characteristics of the array in terms of the broadside peak gains, embedded element patterns (pattern of element 5,5 when all other elements are terminated with $50 \Omega$ loads), and finite array gain patterns are presented in this section.

\section{1) Peak Gains}

The broadside peak gains were calculated using the following equation:

$$
\text { Realized Gain }=4 \pi \mathrm{NA}\left(1-|\Gamma|^{2}\right) / \lambda^{2}
$$

Where $\mathrm{N}$ is the total number of elements; $\mathrm{A}$ is the area of the unit cell; $\Gamma$ is the active (all elements excited) or passive (one element excited and the rest terminated in their input impedance) reflection coefficient; and $\lambda$ is the wavelength.

The ideal broadside peak gain is obtained when the reflection coefficient is assumed to be zero. Referring to Fig. 15, the broadside peak gains for the simulated infinite array and the measured results (obtained from the measured active reflection coefficient) agree very closely to the ideal gain when $\mathrm{N}=100$. 
The result for the simulated finite array with 60 excited elements also tracks very closely to the ideal case when $\mathrm{N}=60$.

The passive reflection coefficient, $\Gamma_{5}, 5$, was also measured from which the broadside peak gain was calculated. This gain

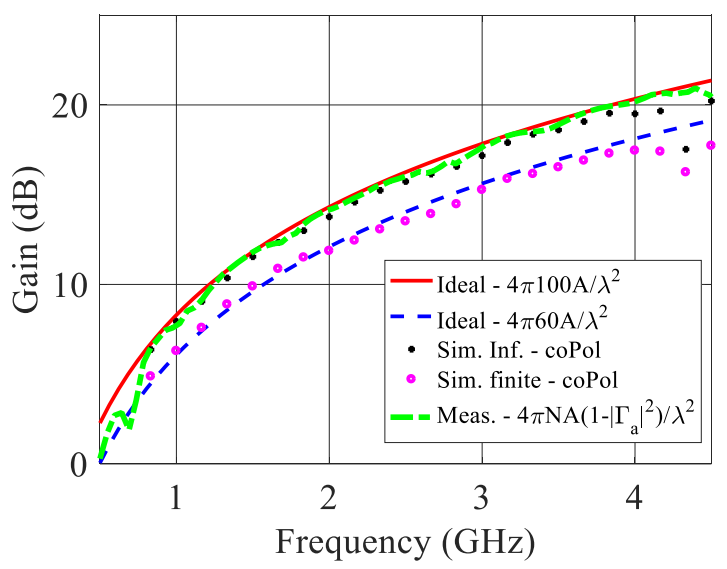

Fig. 15. Measured (obtained from the measured active reflection coefficient) and simulated broadside peak gains of the whole array. The finite array simulation is for 60 active elements with the first two and last two columns of the array terminated in $50 \Omega$ loads.

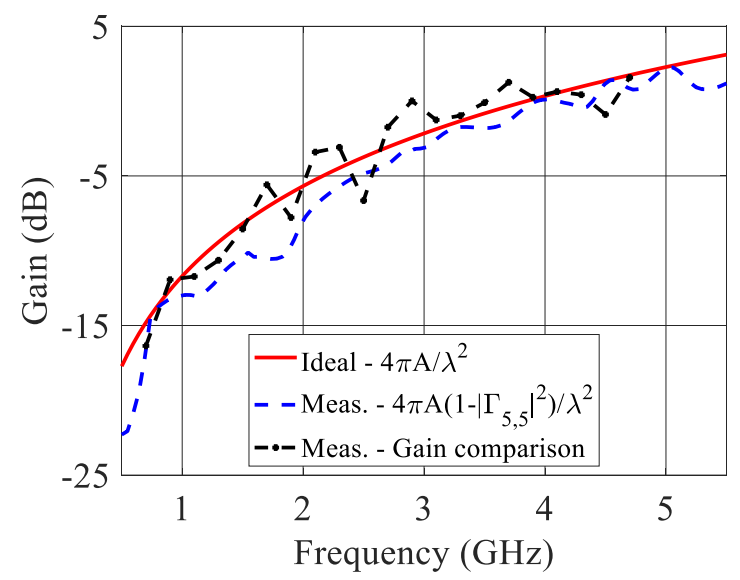

Fig. 16. Measured (obtained from the measured passive reflection coefficient and from the gain comparison method) and ideal broadside peak gains of element 5,5 with all other elements terminated in $50 \Omega$ loads.

is compared to the measured gain in the anechoic chamber using a standard gain antenna (gain comparison method). The results obtained from the gain comparison method includes all the mismatch and radiation losses unlike the reflection coefficient method which only contain the mismatch losses. The results of these measurements are shown in Fig. 16 together with the ideal case. The worst-case deviation from the ideal curve is within $2.5 \mathrm{~dB}$ which occurs at $1.8 \mathrm{GHz}$ for the reflection coefficient method and at $2.5 \mathrm{GHz}$ and $2.9 \mathrm{GHz}$ for the gain comparison method. These discrepancies are attributed to the small array size and the lack of absorbing material around the cables below the array ground. The copper tape used to hold the antenna cards upright and to enable soldering onto the aluminum ground plane also causes resistive losses resulting in higher than expected gains at certain frequencies. For the rest of the frequency points, there is a high degree of agreement.

\section{2) Embedded Element Patterns}

The embedded element patterns of element 5, 5 were measured in an anechoic chamber and the results compared with simulations. The element patters at $4.1 \mathrm{GHz}$ across the $\mathrm{E}, \mathrm{H}$,

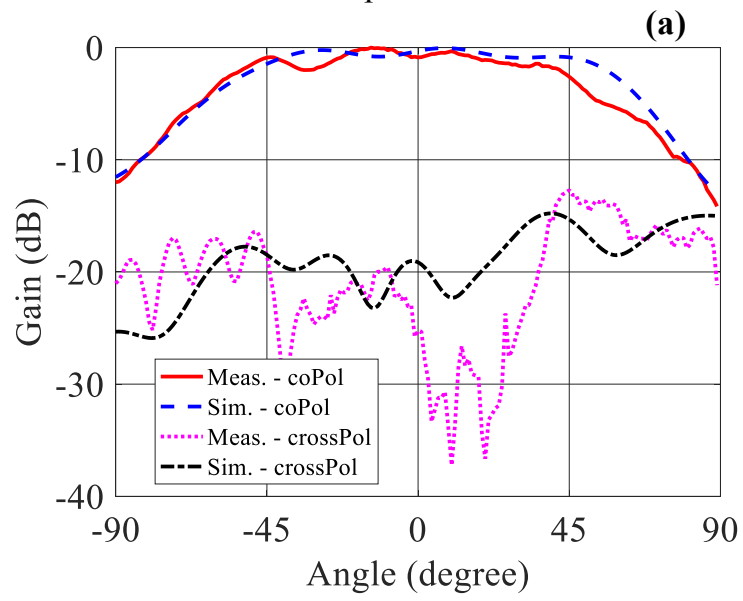

(b)

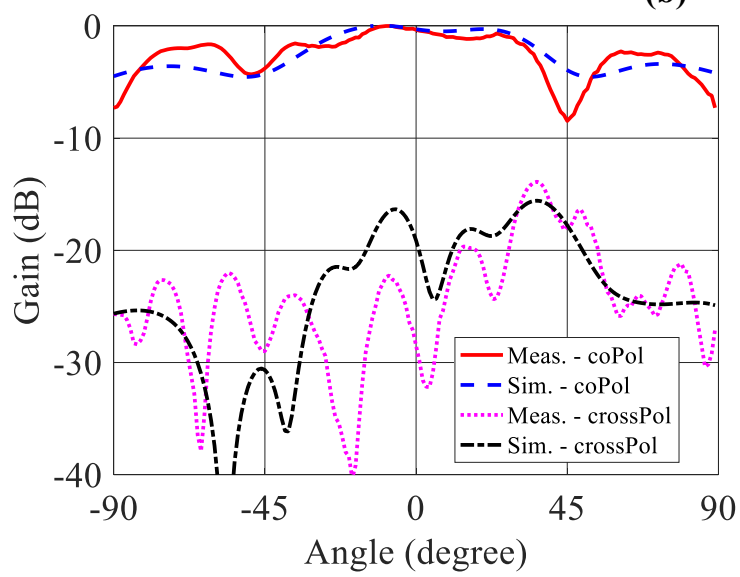

(c)

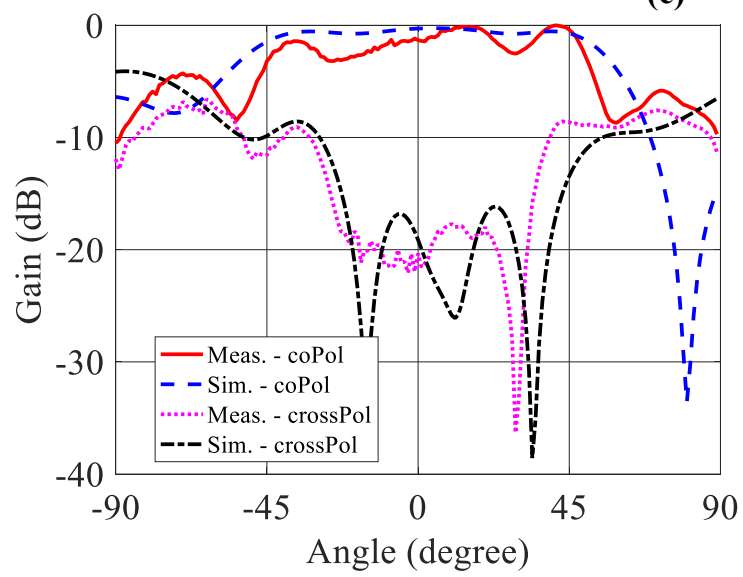

Fig. 17. Embedded element patterns at $4.1 \mathrm{GHz}$. (a) E-plane. (b) H-plane. (c) D-plane.

and D-planes is shown in Fig. 17. The simulated and measured results track very well with each other.The small amount of discrepancies is mainly attributed to fabrication imperfections, the small size of the array and ground plane, edge effects, and reflections from the feed cables. 


\section{3) Finite Array Patterns}

The scanning ability of the array across the E, H, and D-planes, at $4.1 \mathrm{GHz}$ are displayed in Fig. 18. During $\mathrm{E}$ and H-plane scans, the cross-polarization levels improves with scan as was noted

(a)

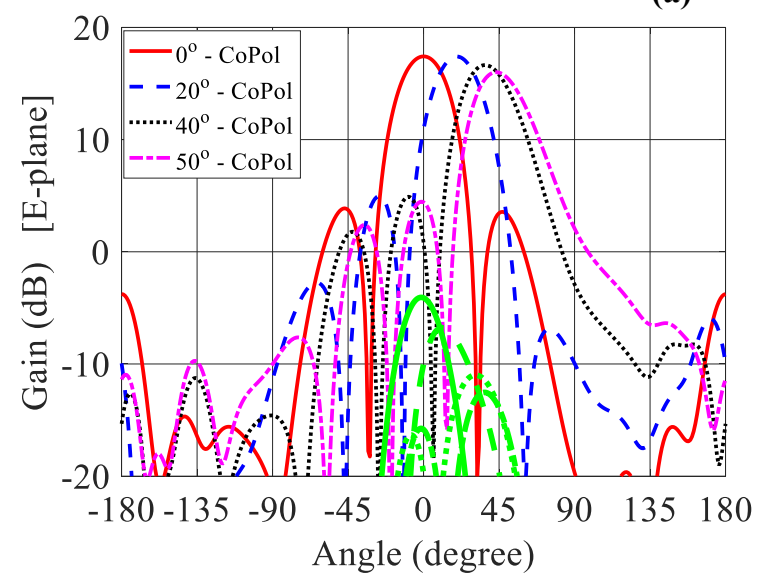

(b)

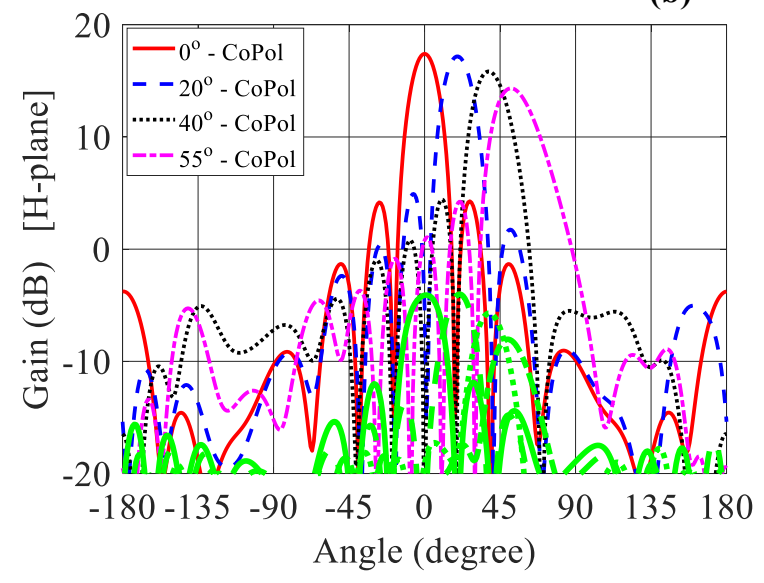

(c)

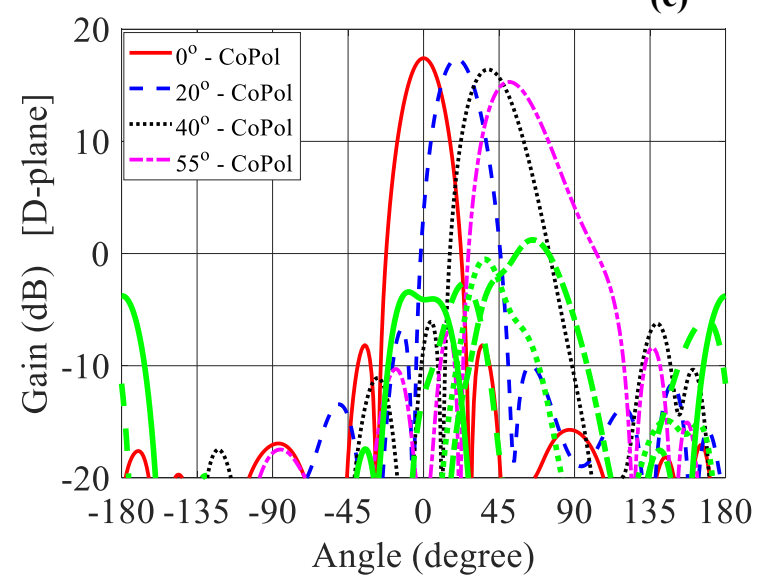

Fig. 18. Finite array gain patterns at $4.1 \mathrm{GHz}$. The patterns are symmetric for both positive and negative scans but only the positive scans are shown for clarity. The cross polarizations are shown using the green curves. (a) E-plane. (b) H-plane. (c) D-plane.

in the infinite array simulations. The difference between the coand cross-polarization levels is $>21 \mathrm{~dB}$ for the $\mathrm{E}$ and H-planes, and $>15 \mathrm{~dB}$ for the $\mathrm{D}$-plane. The peak broadside gain is 17.42 $\mathrm{dB}$. The maximum scan loss varies from $1.93 \mathrm{~dB}$ along the E-plane to $3.3 \mathrm{~dB}$ along the $\mathrm{H}$-plane for a maximum scan angle of $55^{\circ}$. The patterns in the negative scan direction are a mirror

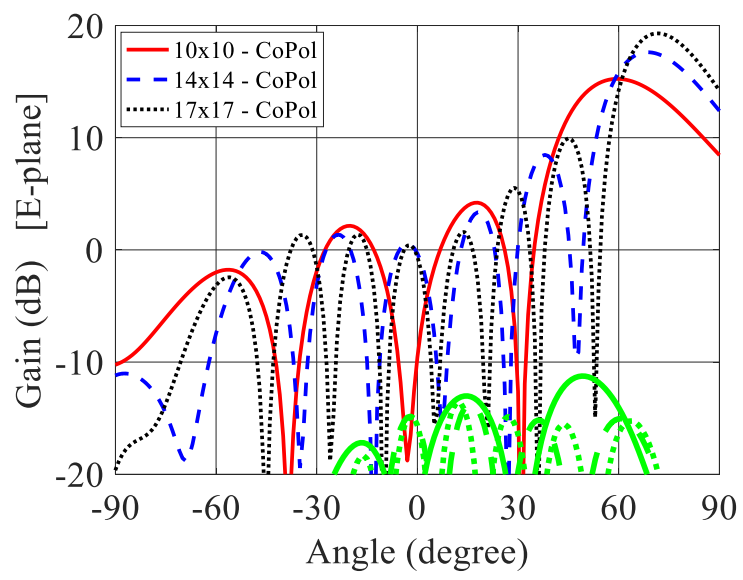

Fig. 19. Simulated E-plane gain patterns pointing at $70^{\circ}$ for the $0.254 \mathrm{~mm}$ thick MS-WAIM design at $4.1 \mathrm{GHz}$. The beam pointing accuracy is shown to improve as the array size is increased from 10x10 to $17 \times 17$ elements. The cross polarizations are shown using the green curves.

image of those in the positive direction. For clarity, only the positive scan patterns are shown.

In order to demonstrate the widest scan range of the 0.254 $\mathrm{mm}$ thick MS-WAIM design, its E-plane gain pattern for $70^{\circ}$ scan is simulated. The edge elements along the E-plane were also terminated in this case in order to obtain a wideband impedance match. The resulting narrower array size causes the beam to spread which leads to an increase in the beam-pointing error for wider scan angles. This behavior corresponds to the fact that the beam pointing error will increase as the array size decreases [36]. To improve the wide angle beam pointing accuracy, the array size was increased from 10x10 elements to $17 \times 17$ elements. The consequent beam scanning results for the different array sizes are displayed in Fig. 19. It can be seen that the $17 \times 17$ array element system provides the desired beam pointing accuracy at $70^{\circ}$.

\section{DISCUSSION}

To quantify the implication of our work in terms of fundamental limits and in comparison to other wideband PEC-backed antenna arrays, the array figure of merit [22] is utilised. The array figure of merit is a single number which takes into account the bandwidth, scan range, and total efficiency, for a given array height. The total efficiency is a combination of the radiation and mismatch efficiencies. According to [22], the definition of the array figure of merit depends on whether the array is lossy or if it has negligible losses. For arrays with negligible losses:

$$
\mathrm{P}_{\mathrm{A}}=\mathrm{B} \log \left(1 / \Gamma_{\max }\right) / \cos \theta_{\max }
$$

Where $\mathrm{B}=\left(\omega_{\max }-\omega_{\min }\right) / \sqrt{ }\left(\omega_{\max } \omega_{\min }\right), \theta_{\max }$ is the maximum scan angle, $\Gamma_{\max }$ is the worst-case reflection coefficient, and $\log$ is the natural logarithm. For lossy arrays:

$$
\mathrm{P}_{\mathrm{A}}=\mathrm{B} \mid \log \left(1-\eta_{\min }\right) / 2 \cos \theta_{\max }
$$


Where $\eta_{\min }$ is the total efficiency. The total efficiency of the array with the $0.254 \mathrm{~mm}$ and the $0.508 \mathrm{~mm}$ superstrates are displayed in Fig. 20. The total efficiency values are shown for broadside and for the widest scan angles. The broadside total

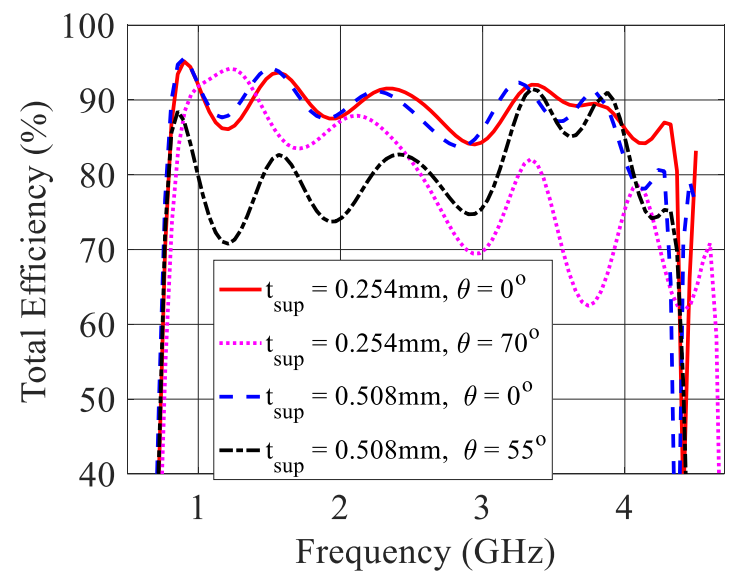

Fig. 20. The total efficiency of the array with the $0.254 \mathrm{~mm}$ and the $0.508 \mathrm{~mm}$ superstrates. Total efficiency values are shown for broadside and for the widest scan angles.

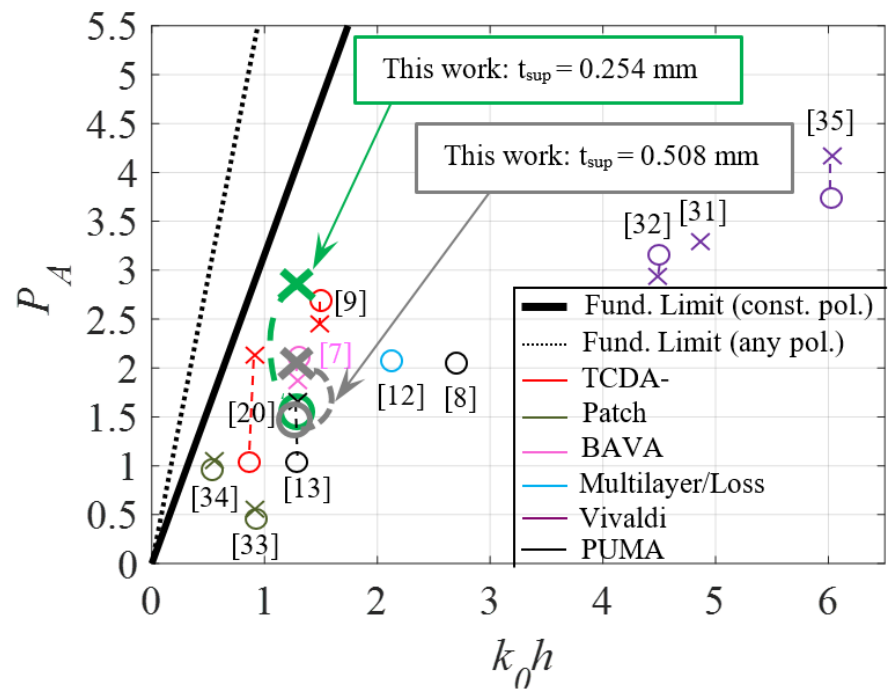

Fig. 21. Performance comparison of wideband PEC-backed antenna arrays using the array figure of merit $\left(\mathrm{P}_{\mathrm{A}}\right)$ versus electrical thickness $\left(\mathrm{k}_{0} \mathrm{~h}\right)$ plot. This figure is reproduced here courtesy of the work done in [22] with the addition of some recently reported works. The current work is shown in bold green and bold grey. The circles represent broadside performance and the crosses represent scanning performance along the $\mathrm{E}$ or $\mathrm{H}$ planes.

TABLE III

COMPUTED $\mathrm{P}_{\mathrm{A}}$ AND $\mathrm{K}_{0} \mathrm{H}$ VALUES

\begin{tabular}{|l|l|l|l|l|l|l|l|}
\hline \multirow{2}{*}{$t_{\text {sup }}(\mathrm{mm})$} & $\begin{array}{l}f_{\min } \\
(\mathrm{GHz})\end{array}$ & $\begin{array}{l}f_{\max } \\
(\mathrm{GHz})\end{array}$ & $B$ & $\eta_{\min }$ & $\begin{array}{l}\theta_{\max } \\
\left({ }^{\circ}\right)\end{array}$ & $P_{A}$ & $k_{0} h$ \\
\hline \multirow{2}{*}{$0.254 \mathrm{~mm}$} & 0.80 & 4.38 & 1.91 & 0.80 & 0 & 1.54 & 1.33 \\
\cline { 2 - 8 } & 0.79 & 4.63 & 2.01 & 0.62 & 70 & 2.84 & 1.36 \\
\hline \multirow{2}{*}{$0.508 \mathrm{~mm}$} & 0.79 & 4.32 & 1.91 & 0.78 & 0 & 1.45 & 1.31 \\
\cline { 2 - 8 } & 0.76 & 4.36 & 1.98 & 0.7 & 55 & 2.08 & 1.29 \\
\hline
\end{tabular}

efficiency is $>78 \%$ for both designs. For the $0.254 \mathrm{~mm}$ design, the total efficiency is $>62 \%$ for scan angles up to $70^{\circ}$. For the
$0.508 \mathrm{~mm}$ design on the other hand, the total efficiency is $>70$ $\%$ for scan angles up to $55^{\circ}$.

Equation (4) was used to calculate the figure of merit and the electrical thickness, $k_{0} h$, where $k_{0}$ is the free space wave number and $h$ is the array height measured from the array ground plane to the top of the array at the centre frequency $\omega_{0}=\sqrt{ }\left(\omega_{\text {low }} \omega_{\text {high }}\right)$. The calculated results for the $0.254 \mathrm{~mm}$ and $0.508 \mathrm{~mm}$ superstrates are shown in table III and plotted in Fig. 21 in bold green and grey respectively. The circles represent broadside performance and the crosses represent scanning performance along the $\mathrm{E}$ or $\mathrm{H}$ planes. From Fig. 21, the $\mathrm{P}_{\mathrm{A}}$ values are 2.84 and 2.08 for the array with the $0.254 \mathrm{~mm}$ and $0.508 \mathrm{~mm}$ superstrates, respectively. The $\mathrm{P}_{\mathrm{A}}$ value of 2.84 is higher than most of the designs reported to date.

\section{CONCLUSION}

This paper presents a wideband antenna array with an integrated low-profile balun. The balun is simple and compact facilitating an overall array height of just $0.088 \lambda_{\mathrm{L}}$. For the optimum design, a bandwidth of 5.5:1 was achieved while scanning to $70^{\circ}$ in the E-plane and $55^{\circ}$ in the H-plane. In addition, the fact that the balun and dipoles are printed on the same substrate, ensures simplicity in construction and cost reduction. Due to the unique ability of the MS-WAIM to improve the impedance matching as the scan angle increases, the resulting cross-polarization levels decreases instead of increasing as is usually the case. A $10 \times 10$ array prototype was fabricated and tested. The measured and simulated results agree quite well. For larger array sizes, the radiation and matching characteristics will improve even further.

\section{ACKNOWLEDGMENT}

The authors would like to thank the staff at the Faculty of Engineering and Information Technology (FEIT) workshop of the University of Technology Sydney, and the staff at Jenkins Engineering Defense Systems (JEDS) for their help with the array fabrication and measurement respectively.

\section{REFERENCES}

[1] G. C. Tavik, C. L. Hilterbrick, J. B. Evins, J. J. Alter, J. G. Crnkovich, J.W. De Graaf, W. Habicht, G. P. Hrin, S. A. Lessin, D. C. Wu, and S. M. Hagewood, "The advanced multifunction RF concept," IEEE Trans. Microwave Theory Techn., vol. 53, no. 3, pp. 1009-1020, Mar. 2005.

[2] P. S. Hall, P. Gardner, and A. Faraone, "Antenna Requirements for Software Defined and Cognitive Radios," in Proc. of the IEEE, vol. 100, no. 7, pp. 2262-2270, July 2012.

[3] W. F. Croswell, T. Durham, M. Jones, D. Schaubert, P. Friederich, and J. G. Maloney, "Wideband arrays," in Modern Antenna Handbook, C.A. Balanis, Ed. New York: Wiley- Interscience, pp. 581-627, 2008.

[4] R. W. Kindt, W. R. Pickles, "Ultrawideband all-metal flared-notch array radiator," IEEE Trans. Antennas Propag., vol. 58, no. 11, pp. 3568 - 3575, Sept. 2010.

[5] R. Kindt and R. Pickles, "12-to-1 bandwidth all-metal Vivaldi array element," in Proc. IEEE Antennas and Propag. Soc. Int. Symp., Charleston, SC, 2009, pp. 1-4.

[6] D. T. McGrath, N. Schuneman, T. H. Shively and J. Irion, "Polarization properties of scanning arrays," in Proc. IEEE Int. Symp. on Phased Array Systems and Technology, 2003, pp. 295-299.

[7] M.W. Elsallal and J. C. Mather, "An ultra-thin, decade (10:1) bandwidth, modular "BAVA" array with low cross-polarization," in Proc. IEEE Int. Symp. Antennas Propag. (APSURSI'11), July 2011, pp. 1980-1983. 
[8] B. Munk, et al., "A low-profile broadband phased array antenna," in Proc. IEEE Antennas Propag. Soc. Int. Symp. (APS'03), Columbus, $\mathrm{OH}$ USA, Jun. 2003, vol. 2, pp. 448-451.

[9] J. P. Doane, K. Sertel, and J. L. Volakis, "A Wideband, Wide Scanning Tightly Coupled Dipole Array With Integrated Balun (TCDA-IB)," IEEE Trans. Antennas Propag., vol. 61, no. 9, pp. 4538-4548, Sep. 2013.

[10] D. Cavallo, A. Neto, G. Gerini, A. Micco and V. Galdi, "A 3- to 5-GHz Wideband Array of Connected Dipoles with Low Cross Polarization and Wide-Scan Capability," IEEE Trans. Antennas Propag., vol. 61, no. 3, pp. 1148-1154, Mar. 2013.

[11] R. C. Hansen, "Linear Connected Arrays," IEEE Antennas Wireless Propag. Lett., vol. 3 pp. 154-156, 2004.

[12] W. F. Moulder, K. Sertel, and J. L. Volakis, "Superstrate-enhanced ultrawideband tightly coupled array with resistive FSS," IEEE Trans. Antennas Propag., vol. 60, no. 9, pp. 4166-4172, 2012.

[13] S. S. Holland and M. N. Vouvakis, "The Planar Ultrawideband Modular Antenna (PUMA) Array," IEEE Trans. Antennas and Propag., vol. 60, no. 1, pp. 130-140, Jan. 2012.

[14] S. S. Holland, D. H. Schaubert, and M. N. Vouvakis, "A 7-21 GHz Dual-Polarized Planar Ultrawideband Modular Antenna (PUMA) Array," IEEE Trans. Antennas Propag., vol. 60, no. 10, pp. 4589-4600, Oct. 2012.

[15] A. Oliner and G. Knittel, Eds., "Phased array antennas," in Proc. 1970 Phased Array Symposium, 1972 (Artech House, Dedham, MA).

[16] E. G. Magill and H. A. Wheeler, "Wide-angle impedance matching of a planar array antenna by a dielectric sheet," IEEE Trans. Antennas Propag., vol. 14, no. 1, pp. 49-53, Jan. 1966.

[17] S. Lee and R. Mittra, "Radiation from dielectric-loaded arrays of parallel-plate waveguides," IEEE Trans. Antennas Propag., vol. 16, no. 5, pp. 513-519, Sep. 1968

[18] S. Sajuyigbe, M. Ross, P. Geren, S. A. Cummer, M. H. Tanielian, and D. R. Smith, "Wide angle impedance matching metamaterials for waveguide-fed phased-array antennas," IET Microw. Antennas Propag., vol. 4, no. 8, pp. 1063-1072, Aug. 2010.

[19] T. R. Cameron and G. V. Eleftheriades, "Analysis and characterization of a wide-angle impedance matching metasurface for dipole phased arrays," IEEE Trans. Antennas Propag., vol. 63, no. 9, pp. 3928-3938, Sep. 2015.

[20] E. Yetisir, N. Ghalichechian and J. L. Volakis, "Ultrawideband array with $70^{\circ}$ scanning using FSS superstrate," IEEE Trans. Antennas Propag., vol. 64, no. 10, pp. 4256-4265, Oct. 2016.

[21] A. O. Bah, P. Y. Qin, R. W. Ziolkowski, Q. Cheng and Y. J. Guo, "Realization of an Ultra-thin Metasurface to Facilitate Wide Bandwidth, Wide Angle Beam Scanning," Scientific Reports, vol. 8, no. 1, pp. 4761, Mar. 2018.

[22] J. P. Doane, "Wideband low-profile antenna arrays: Fundamental limits and practical implementations," Ph.D. dissertation, Dept. Elect. Comput. Eng., Ohio State Univ., Columbus, OH, USA, 2013.

[23] Z. Szabo, G. H. Park, R. Hedge and E. P. Li, "A unique extraction of metamaterial parameters based on Kramers-Kronig relationship," IEEE Trans. Microw. Theory Techn., vol. 58, no. 10, pp. 2646-2653, Oct. 2010.

[24] R. W. Klopfenstein, "A Transmission Line Taper of Improved Design," in Proc. of the IRE, vol. 44, Jan. 1956, pp. 31-15.

[25] Matlab R2016, The Mathworks, Inc., Natick, MA [Online]. Available: https://www.mathworks.com/products/matlab/

[26] D. M. Pozar, Microwave Engineering, ch. 3, pp. 148-149, John Wiley \& Sons, Hoboken, NJ, 2012.

[27] ANSYS HFSS ver. 16, ANSYS, Inc., Canonsburg, PA [Online]. Available: https://www.ansoft.com/products/hf/hfss/

[28] R. J. P. Douville and D. S. James, "Experimental Characterization of Microstrip Bends and Their Frequency Dependent Behavior," in Proc. IEEE Conf. Digest, Oct. 1973, pp. 24-25.

[29] A. Ludwig, "The definition of cross polarization," IEEE Trans. Antennas Propag., vol. 21, pp. 116-119, Jan. 1973.

[30] M. N. Vouvakis and D. H. Schaubert, "Vivaldi antenna arrays," in Chapter 3 in Frontiers in Antennas: Next Generation Design Engineering, F. B. Gross, Ed. New York: McGraw-Hill, 2011.

[31] H. Holter, T.H. Chio, and D. H. Schaubert, "Experimental results of 144-element dual-polarized endfire tapered-slot phased arrays," IEEE Trans. Antennas Propag., vol. 48, no. 11, pp. 1707-1718, Nov. 2000.

[32] D. H. Schaubert, S. Kasturi, A. O. Boryssenko, and W. M. Elsallal, "Vivaldi antenna arrays for wide bandwidth and electronic scanning," in Proc. EuCAP - European Conference on Antennas \& Propagation. IET, Nov. 2007, pp. 1-6.
[33] M. J. Buckley, J. Wolf, B. J. Herting, J. Mather, D. Manson, and J. B. West, "Wide band and wide scan metamaterial loaded radiating elements," in Proc. Antennas and Applications Symposium, Allerton IL, Sep. 2010.

[34] R. Erickson et al., "Wideband and wide scan phased array microstrip patch antennas for small platforms," in Proc. EuCAP - European Conference on Antennas \& Propagation. IET, Nov. 2007, pp. 1-6.

[35] M. Stasiowski and D. Schaubert, "Broadband array antenna," in Proc. Antennas and Applications Symposium, Allerton IL, Sep. 2008.

[36] R. C. Hansen, Phased Array Antennas, ch. 15, pp. 526-527, John Wiley \& Sons, Hoboken, NJ, 2009.

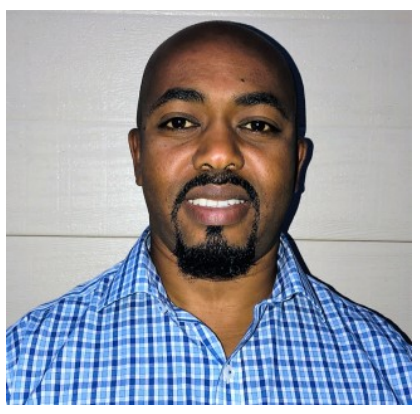

Alpha O. Bah (S'15) received his undergraduate training in Electrical and Electronic Engineering from Institut Teknologi Brunei (ITB), Brunei Darussalam, in 1998. From 1998 to 2014, he worked in Telecommunications for various companies around Australia including Optus, Telstra, Xstrata, and Thiess. His responsibilities included design, installation, commissioning, and testing of Telecommunications networks.

In 2014, He obtained his MEng. Degree in Networking and Communications from the Queensland University of Technology, Brisbane, Australia. Since 2015, he has been pursuing his PhD in ultrawideband antenna arrays at the University of Technology Sydney. His research interests include ultrawideband antenna arrays, metasurface enhanced antennas, Fabry-Perot antennas, and $\mathrm{mm}$-wave antennas. 